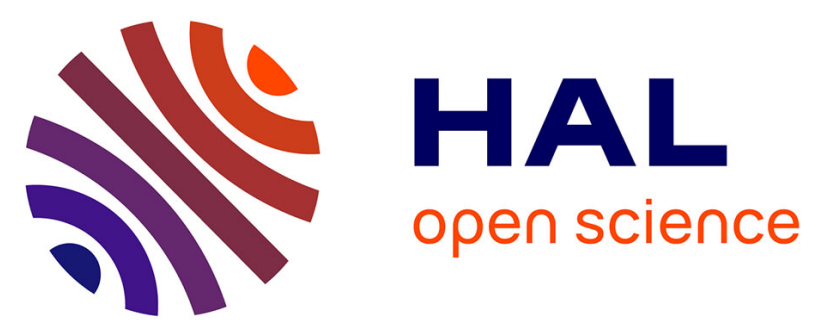

\title{
Estimation and Stabilization of Humanoid Flexibility Deformation Using Only Inertial Measurement Units and Contact Information
}

\author{
Mehdi Benallegue, Florent Lamiraux
}

\section{- To cite this version:}

Mehdi Benallegue, Florent Lamiraux. Estimation and Stabilization of Humanoid Flexibility Deformation Using Only Inertial Measurement Units and Contact Information. International Journal of Humanoid Robotics, 2015, 2014 IEEE-RAS International Conference on Humanoid Robots, 12 (3), pp.15500255. 10.1142/S0219843615500255 . hal-01169149v2

\author{
HAL Id: hal-01169149 \\ https://hal.science/hal-01169149v2
}

Submitted on 26 Oct 2018

HAL is a multi-disciplinary open access archive for the deposit and dissemination of scientific research documents, whether they are published or not. The documents may come from teaching and research institutions in France or abroad, or from public or private research centers.
L'archive ouverte pluridisciplinaire $\mathbf{H A L}$, est destinée au dépôt et à la diffusion de documents scientifiques de niveau recherche, publiés ou non, émanant des établissements d'enseignement et de recherche français ou étrangers, des laboratoires publics ou privés. 


\title{
ESTIMATION AND STABILIZATION OF HUMANOID FLEXIBILITY DEFORMATION USING ONLY INERTIAL MEASUREMENT UNITS AND CONTACT INFORMATION
}

\author{
Mehdi Benallegue*, Florent Lamiraux*
}

October 26, 2018

\begin{abstract}
Most robots are today controlled as being entirely rigid. But often, as for HRP-2 robot, there are flexible parts, intended for example to absorb impacts. The deformation of this flexibility modifies the orientation of the robot and endangers balance. Nevertheless, robots have usually inertial sensors (IMUs) to reconstruct their orientation based on gravity and inertial effects. Moreover, humanoids have usually to ensure a firm contact with the ground, which provides reliable information on surrounding environment. We show in this study how important it is to take into account these information to improve IMU-based position/orientation reconstruction. We use an extended Kalman filter to rebuild the deformation, making the fusion between IMU and contact information, and without making any assumption on the dynamics of the flexibility. We show how, with this simple setting, we are able to compensate for perturbations and to stabilize the end-effector's position/orientation in the world reference frame. We show also that this estimation is reliable enough to enable a closed-loop stabilization of the flexibility and control of the CoM position with the simplest possible model.
\end{abstract}

balance; compliance; state observation; sensor fusion; inertial measurement unit; force sensors; stabilization

\section{PROBLEM STATEMENT}

Many current humanoid robots are controlled as rigid systems, even if there are compliant and flexible parts in it. A good example of such a system is the robot HRP-2. Between the ankle and the sole of the robot, there lies a flexible bush (see Fig 1), designed to absorb foot impacts in order to protect force sensors and leg actuators[1]. However, this flexible part acts also as an angular spring and generates important deviation of the whole body, including the center of mass (CoM), which is not modeled in the rigid system.

Therefore, this flexibility can threaten the balance of the robot, for example if the deformation deviates the CoM enough. Moreover, it may also jeopardize environment-related tasks. For example, in the case of drilling a wall, a robot has to apply forces on the wall. These forces will create a deformation of the compliant material and will deviate the robot's tool from its reference position and/or orientation.

The problem of HRP-2 flexibility is currently tackled by a robot stabilizer. The stabilizer drives the deformation of the flexible material to produce the desired forces and moments at feet, using a model of elasticity of the flexible material[2,3]. At the same time, the upper-body orientation is maintained vertical, in order to minimize unwanted angular momentum and to enable to respect orientation-related upper-body tasks (manipulation, gaze, etc.). The orientation of the

${ }^{*}$ CNRS, LAAS, 7 avenue du colonel Roche, F-31400 Toulouse, France mehdi.benallegue@laas.fr, florent@laas.fr 


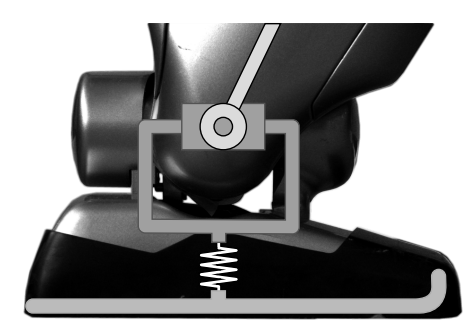

Figure 1: The foot of HRP-2. Between the ankle joint and the sole of the robot, there is a rubber bush.

upper body is obtained using an inertial measurement unit at the chest of the robot. However, with this stabilizer, in the case of upper-body tasks, even if the torso is maintained upright, there is no guarantee that upper-body limbs are at their reference position, which is usually important when the robot is interacting with the environment. Moreover, the use of controllers based on force/torque sensors raises two main issues. First, these solutions are sensitive to sensors' calibration errors[4], which can even appear during operation of the robot (e.g. due to impacts, under constraints, etc). Second, these approaches can obviously not apply to robots which are not equipped with these expensive force sensors at contact points, such as Aldebaran's Romeo robot[5].

By contrast, inertial measurement sensors are cheap, relatively reliable and more robust. In addition, they provide important data on the real kinematics of the robot in the inertial frame. We show in this paper that, if we couple these sensors with contact point positions, we can afford a real-time fine estimation of the flexibility state, without any model of its compliance dynamics. We show also that these measurements can enable a stabilization of the robot's end-effector in the presence of external perturbations. Finally, we demonstrate that this flexibility estimation is reliable enough to drive a stable closed-loop second order control of the robot's center of mass in order to stabilize the flexibility and avoid robot's oscillation.

This study aims at proving the efficiency of inertial sensors for whole body pose estimation, and perturbations detection. In the next section, we describe the theoretical guarantees and improvements provided by contact information to IMU measurements. Section III shows an example of implementation of such an observer with minimum prior knowledge. Section IV presents an experimental setting where we use our method for end-effector stabilization and compensation of perturbations. Section V demonstrate that a stable closed loop flexibility stabilizer can use the state feedback of our observer. Experimental results of this stabilization are showed afterwards. Finally, Section VI concludes the paper.

\section{THEORETICAL GROUNDING}

In the case of humanoid locomotion, the contact forces have usually to respect center of pressure $(\mathrm{CoP})$ and friction cone constraints in order to maintain balance[6]. That means that the contacts are firmly fixed to the environment. However, most methods for humanoid-robots attitudeestimation using inertial measurements do not consider contacts information, even for stabilizing the robots on their feet $[7,8]$. Fixed contact positions provide coupling between the rotation and translation, transforming the inertial measurement unit (IMU) into a much more efficient sensor for reconstructing position/orientation. We show next a simple example of what this coupling provides to a simple pendulum.

\subsection{The inverted pendulum}

Let's consider a simple 3D inverted pendulum of $1 \mathrm{~m}$ length, connected to the ground with a 3 degrees of freedom (DoF) ball joint, and with an IMU, rigidly aligned at the top of the pendulum 
(see Fig. 2). The configuration of the pendulum is the rotation matrix $R$. This orientation defines the position $\mathbf{p}$ of the IMU in the global frame:

$$
\mathbf{p}=R \mathbf{e}_{\mathbf{z}}
$$

where $\mathbf{e}_{\mathbf{z}}=\left[\begin{array}{lll}0 & 0 & 1\end{array}\right]^{t}$ is the unit vector along vertical $\mathbf{z}$ axis. If we consider that an unknown external source $\mathbf{u}$ provides the control of the pendulum's acceleration, we can write the following state dynamics:

$$
\dot{\mathbf{x}}=\left[\left([\omega]_{\times} R\right)^{t} \quad \dot{\omega}^{t} \quad 0\right]^{t}+\left[\begin{array}{lll}
0 & 0 & I
\end{array}\right]^{t} \mathbf{u},
$$

where $\mathbf{x}=\left[\begin{array}{lll}R^{t} & \omega^{t} & \dot{\omega}^{t}\end{array}\right]^{t}$ is the state vector formed by orientation, the angular velocity vector and the angular acceleration ${ }^{1}$, and $[\cdot]_{\times}$is the skew symmetric operator, i.e.:

$$
\left[\begin{array}{l}
x \\
y \\
z
\end{array}\right]_{\times}=\left[\begin{array}{ccc}
0 & -z & y \\
z & 0 & -x \\
-y & x & 0
\end{array}\right]
$$
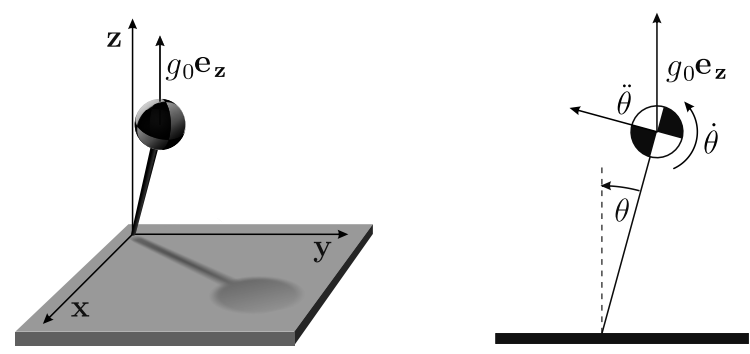

Figure 2: On the left, 3D inverted pendulum. On the right, 2D inverted pendulum

The IMU at the top is composed of a gyrometer providing the angular rate $\mathbf{y}_{\mathbf{g}}$ and an accelerometer providing the gravity and the linear acceleration $\mathbf{y}_{a}$. Let $\mathbf{y}=\left[\begin{array}{ll}\mathbf{y}_{g}^{t} & \mathbf{y}_{a}^{t}\end{array}\right]^{t}$ be the whole measurements vector:

$$
\mathbf{y}=\left[\begin{array}{c}
R^{t} \omega \\
R^{t}\left(\ddot{\mathbf{p}}+g_{0} \mathbf{e}_{\mathbf{z}}\right)
\end{array}\right]
$$

with $g_{0}$ the standard gravity constant. This measurement provides partial data on the configuration, but we show next that observable parts of the vector differ significantly depending on whether we consider the contact coupling or not.

\subsection{Without translation/rotation coupling}

In the case we do not take into account the coupling, we have to consider that the linear acceleration $\ddot{\mathrm{p}}$ is a free input to the system for which we do not have any model. So, let's consider the case of an input acceleration $\ddot{\mathbf{p}}=-g \mathbf{e}_{\mathbf{z}}$, we have then $\mathbf{y}_{a}^{t}=0$, and the measurements provide only angular velocities, which are insufficient to reconstruct the orientation. Therefore, the configuration of the pendulum itself is not observable with these hypotheses.

Indeed, most today's approaches to reconstruct orientations with a gyrometer/accelerometer sensors without contact information, have to put an erroneous model on the linear acceleration, for example considered as Gaussian white noise for Kalman Filtering [9] or as high-frequency signal for complementary filtering[10]. These method lead to ignore translation accelerations, which may carry important and redundant information on the dynamics of the pendulum.

\footnotetext{
${ }^{1}$ For simplicity, we keep a matrix representation of the orientation despite the heterogeneous nature of this state vector, in the next section we use vector representation for the actual implementation.
} 


\subsection{With translation/rotation coupling}

If we use the rotation/translation coupling provided by Equation (1), we have then

$$
\mathbf{y}=\left[\begin{array}{c}
R^{t} \omega \\
{\left[R^{t} \dot{\omega}\right]_{\times} \mathbf{e}_{\mathbf{z}}+\left[R^{t} \omega\right]_{\times}^{2} \mathbf{e}_{\mathbf{z}}+g_{0} R^{t} \mathbf{e}_{\mathbf{z}}}
\end{array}\right]
$$

and let's consider the first derivative of the gyrometer measurements

$$
\dot{\mathbf{y}}_{g}=R^{t} \dot{\omega}
$$

with these three vectors $\mathbf{y}_{g}, \mathbf{y}_{a}$ and $\dot{\mathbf{y}}_{g}$, we can reconstruct $R^{t} \mathbf{e}_{\mathbf{z}}$ and then the roll and pitch components of the configuration $R$. The yaw is unfortunately not observable with these sensors when there is only one contact with the environment, since the system is invariant with respect to rotations around the $\mathbf{z}$ axis.

\subsection{The case of multiple contacts}

If the number of contacts with the environment is 3 or more, the sensor is fully constrained and cannot move. So let's consider the 2 contact situation. There remains only one degree of freedom, which is the rotation around the axis $\left(c_{1} c_{2}\right)$ passing by the two contact points $c_{1}$ and $c_{2}$. The system is equivalent to a $2 \mathrm{D}$ pendulum for which the configuration is defined by one angle $\theta$, and the state becomes $\mathbf{x}=\left[\begin{array}{lll}\theta & \dot{\theta} & \ddot{\theta}\end{array}\right]$ (see Fig. 2). There are two possibilities, (i) the axis $\left(c_{1} c_{2}\right)$ is vertical, and in that case, the sensors can observe only angular velocities and accelerations around this axis, or (ii) the contact points are not vertically aligned and, without loss of generality, we can consider them at the same height and that the IMU is at 1 meter from the axis. If it is not the case, we only need to project the dynamics on the plane orthogonal to the axis $\left(c_{1} c_{2}\right)$ with minor adaptations to find the same developments.

The measurements vector is three-dimensional: angular velocity around the axis $\left(c_{1} c_{2}\right)$, and bi-dimensional accelerations orthogonal to it. All other measurements have constant values, the new measurement vector becomes:

$$
\mathbf{y}=\left[\begin{array}{c}
\dot{\theta} \\
\ddot{\theta}+g_{0} \sin (\theta) \\
\dot{\theta}^{2}+g_{0} \cos (\theta)
\end{array}\right],
$$

which can straightforwardly reconstruct all the state vector.

We see with the pendulum example that rotation/translation coupling, deduced from contact points information, can be taken into account to observe the sensor attitude and position, without resorting to erroneous modeling of the state dynamics. In the next section, we show how we use this idea to reconstruct the state of the flexible part of the robot.

\section{FLEXIBILITY DEFORMATION OBSERVATION}

\subsection{Modeling the flexibility}

HRP-2 is a $30+6$ DoF robot controlled as being rigid. The configuration $\mathbf{q}$ is supposed to define perfectly for each limb $i$ the $6 \mathrm{D}$ position in the world, represented by a homogeneous transformation matrix ${ }^{\mathcal{C}} M_{i}(\mathcal{C}$ superscript is for "control").

However, there is a flexible part in HRP-2 which is a small compliant material between the sole and the ankle joint of the robot[1]. It can be compressed, bent and twisted according to applied forces/moments. Therefore, when the robot is on its feet, the compliance modifies the configuration of the robot in rotation and translation, even when the contacts are balanced and immobile. We depict the flexibility deformation by a $\mathrm{SE}(3)$ transformation represented by a homogeneous transformation matrix

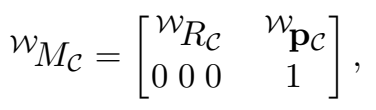


with $\mathcal{W}_{R_{\mathcal{C}}}$ and $\mathcal{W}_{\mathbf{p}_{\mathcal{C}}}$ are the rotation matrix and translation vector associated to the flexibility deformation.

Any limb $i$ at position/orientation ${ }^{c_{M}} M_{i}$ in the "control" reference frame lies in fact at position/orientation $\mathcal{W}_{M_{i}}=\mathcal{W}_{M_{\mathcal{C}}}{ }^{{ }} M_{i}$ in the world actual reference frame (see Fig. 3). Therefore, $\mathcal{W}_{M_{\mathcal{C}}}$ is not the matrix associated to a sole-ankle joint, but a virtual joint between the world and the free-flier root joint of the robot. The choice of this representation is valid for any number of supports and to guarantee continuity regardless of changes in contacts.

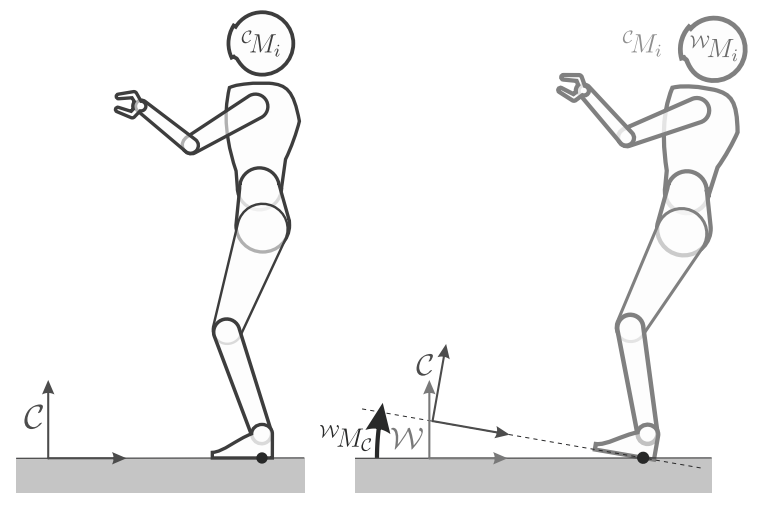

Figure 3: Left, the rigid robot model in the "control" reference frame and the head position at $\mathcal{C}_{M}$. Right, the flexibility $\mathcal{W}_{M_{\mathcal{C}}}$ transforms the configuration of the robot and the real position of the head $\mathcal{W}_{M_{\rangle}}=\mathcal{W}_{M_{\mathcal{C}}}{ }^{c} M_{\rangle}$in the world reference frame. We see that $\mathcal{W}_{M_{\mathcal{C}}}$ is composed of a rotation and a translation.

To simplify notations, we omit the world frame $\mathcal{W}$ upper left superscript for the next developments. Let's then define the 18 dimensional second order state vector $\mathbf{x}=\left[\begin{array}{llllll}\mathbf{p}_{\mathcal{C}}^{t} & \Omega_{\mathcal{C}}^{t} & \dot{\mathbf{p}}_{\mathcal{C}}^{t} & \omega_{\mathcal{C}}^{t} & \ddot{\mathbf{p}}_{\mathcal{C}}^{t} & \dot{\omega}_{\mathcal{C}}^{t}\end{array}\right]^{t}$ where $\Omega_{\mathcal{C}}$ is the rotation vector representation of $R_{\mathcal{C}}$, such that $\exp \left(\left[\Omega_{\mathcal{C}}\right]_{\times}\right)=R_{\mathcal{C}}$, (i.e. $\left\|\Omega_{\mathcal{C}}\right\|$ is the angle or rotation and $\frac{\Omega_{\mathcal{C}}}{\left\|\Omega_{\mathcal{C}}\right\|}$ is the axis) and $\omega_{\mathcal{C}}$ is the vector of angular velocity.

Our study aims at showing that the measurements alone are able to provide accurate estimation of the flexibility state. Hence, we do not model the response of the flexibility to external forces. Instead we take the model of constant acceleration $\ddot{M}_{\mathcal{C}}$, which is a classical choice for pose and attitude estimation[11, 12, 13]. The discrete-time model of the state dynamics is then:

$$
\mathbf{x}_{k+1}=f\left(\mathbf{x}_{k}\right)+\mathbf{v}_{k},
$$

where $f$ is a simple integrator with constant accelerations and $v_{k}$ is Gaussian white noise which is used to model the differences between the real dynamics of the state and the constant-acceleration model.

It is important to note that this dynamical system is chosen only for demonstration purposes, so it is deliberately erroneous and unstable. In fact, for a real use of the proposed observer, we suggest to replace this dynamical model by a model that is closer to the natural dynamics of the studied system, for example by a spring/damper or an inverted pendulum dynamical models. This would increase the precision of the estimation as modeling error is reduced.

\subsection{The sensor system}

This system is not stable and relies only on the measurements to correct it. We use the stock IMU sensor in HRP-2 which is located at the chest, and is composed of an accelerometer and a gyrometer. The measurement vector is then classically:

$$
\mathbf{y}=\left[\begin{array}{c}
\mathbf{y}_{g} \\
\mathbf{y}_{a}
\end{array}\right]=\left[\begin{array}{c}
R_{s}^{t} \omega_{s} \\
R_{s}^{t}\left(\ddot{\mathbf{p}}_{s}+g_{0} \mathbf{e}_{\mathbf{z}}\right)
\end{array}\right]
$$


where $\mathbf{y}_{g}$ and $\mathbf{y}_{a}$ are gyrometer and accelerometer measurements, and $R_{s}, \omega_{s}$ and $\mathbf{p}_{s}$ are respectively the rotation matrix, the angular velocity vector and the position of the IMU in the world reference frame.

We suppose that we know perfectly the robot configuration q and its derivatives, we know then the position ${ }^{\mathcal{C}} R_{s}$, the angular velocity $\mathcal{C}_{\omega_{s}}$, the position ${ }^{\mathcal{C}} \mathbf{p}_{s}$ of the IMU, and their derivatives, in the control reference frame. On the other hand, we have the relationships due to flexibility: $R_{s}=R_{\mathcal{C}}{ }^{\mathcal{C}} R_{s}$ and $\mathbf{p}_{s}=R_{\mathcal{C}} \mathcal{P}_{s}+\mathbf{p}_{\mathcal{C}}$. All that leads to:

$$
\begin{aligned}
& \mathbf{y}_{\mathbf{g}}={ }^{\mathcal{C}} R_{s}^{t} \mathcal{C}_{\omega_{s}}+{ }^{\mathcal{C}} R_{s}^{t} R_{\mathcal{C}}^{t} \omega_{\mathcal{C}} \\
& \mathbf{y}_{\mathbf{a}}={ }^{\mathcal{C}} R_{s}^{t} R_{\mathcal{C}}^{t}\left(\left(\left[\dot{\omega}_{\mathcal{C}}\right]_{\times}+\left[\omega_{\mathcal{C}}\right]_{\times}^{2}\right) R_{\mathcal{C}}{ }^{\mathcal{C}} \mathbf{p}_{s}+2\left[\omega_{\mathcal{C}}\right]_{\times} R_{\mathcal{C}}{ }^{\mathcal{C}_{\mathbf{p}}} \dot{\mathbf{p}}_{s}\right) \\
& +{ }^{\mathcal{C}} R_{s}^{t}\left({ }_{\ddot{\mathbf{p}}_{s}}+R_{\mathcal{C}}^{t} \ddot{\mathbf{p}}_{\mathcal{C}}\right)+g_{0}{ }^{\mathcal{C}} R_{s}^{t} R_{\mathcal{C}}^{t} \mathbf{e}_{\mathbf{z}}
\end{aligned}
$$

Since the measurements are noisy and our dynamics is discretized, we model the sensors dynamics as following

$$
\mathbf{y}_{k}=g\left(\mathbf{x}_{k}, \mathbf{q}_{k}, \dot{\mathbf{q}}_{k}, \ddot{\mathbf{q}}_{k}\right)+\mathbf{w}_{\mathbf{s} k},
$$

where $g$ is the measurement function summarizing equations (10) and (11) and $\mathbf{w}_{\mathbf{s} k}$ is a Gaussian white noise.

\subsection{The contact points}

We have seen that the flexibility provides six supplementary degrees of freedom to the robot. However, the size of the flexible bush and the magnitude of the linear forces exerted on it make linear translations of the compliance almost negligible. In other words, we can consider that globally the contact point positions in the world and in the control reference frame are almost identical: $M_{\mathcal{C}} \mathcal{C}_{c_{i}} \simeq \mathcal{C}_{\mathbf{p}_{i}}$, where $\mathcal{C}_{c_{i}}$ is the position of the $i$-th contact in the control reference frame, which is considered as perfectly known for all the contacts. We put then an approximation instead of an equality constraint. This allows us to leave some freedom to violate the constraint and to detect for example sideways perturbations during double support. Thus, instead of constraining the contact points to be fixed in the environment, we add fake measurements to our dynamical system. These measurement claim that the contacts are fixed in the environment $M_{\mathcal{C}} \mathcal{C}_{c_{i k}}$ $\mathcal{C}_{c_{i k}}=0$, but we model these measurements as being noisy to allow some freedom. So for each contact $c_{i}$, the sensors model is:

$$
\mathbf{r}_{\mathbf{i} k}=M_{\mathcal{C} k} \mathcal{C}_{c_{i k}}-{ }^{\mathcal{C}} \mathbf{p}_{c_{i k}}+\mathbf{w}_{\mathbf{i} k}
$$

where $\mathbf{w}_{\mathbf{i} k}$ is a Gaussian white noise.

We can gather then the measurements of a $n$ contacts configuration in a $6+(3 \times n)$ dimensional vector:

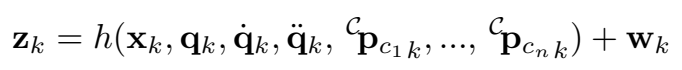

where $h$ stacks all the measurements described earlier (IMU + fake measurements) and $\mathbf{w}_{k}$ stacks all the measurement noises.

It is worth mentioning that, for the continuous-time system, the measurements described here provide the same observability properties as the inverted pendulum example of the previous section. In the case of one contact, the orientation is observable except in yaw, which leads to a partial observability of the position $\mathbf{p}_{\mathcal{C}}$ (constrained to a circle around the vertical line passing by the contact point). In the case of multiple contacts, everything is observable.

\subsection{Extended Kalman Filtering}

We use for the estimation of the state vector a classical Extended Kalman Filter (EKF). This observer works in two steps for each time sample: the prediction and the update. 


\subsubsection{Prediction}

Let's suppose that at instant $k+1$, we have already an estimation of $\mathbf{x}_{k}$ which we denote $\hat{\mathbf{x}}_{k}$. We model the error $\mathbf{e}_{k}=\mathbf{x}_{k}-\hat{\mathbf{x}}_{k}$ as a random variable following a centered Gaussian distribution for which we suppose that we know the covariance matrix $P_{k}$. The prediction consists in simulating the modeled dynamics of the system if it were neither noisy nor perturbed. We define $\overline{\mathbf{x}}_{k+1}$ the predicted state as:

$$
\overline{\mathbf{x}}_{k+1}=f\left(\hat{\mathbf{x}}_{k}\right)
$$

This prediction commits also an error $\overline{\mathbf{e}}_{k+1}=\mathbf{x}_{k+1}-\overline{\mathbf{x}}_{k+1}$ which is due to the transport of $\mathbf{e}_{k}$ by $f$ in addition to the process noise $\mathbf{v}_{k+1}$. We model also the error $\overline{\mathbf{e}}_{k+1}$ as a Gaussian random variable and we linearize $f$ to approximate its covariance matrix:

$$
\bar{P}_{k+1}=F_{k+1} P_{k} F_{k+1}^{t}+Q_{k+1},
$$

where $Q_{k+1}$ is the covariance matrix of $\mathbf{v}_{k+1}$, and

$$
F_{k+1}=\left.\frac{\partial f}{\partial \mathbf{x}}\right|_{\mathbf{x}=\hat{\mathbf{x}}_{k}}
$$

This prediction enables us to estimate the measurements obtained if the state was equal to the prediction:

$$
\overline{\mathbf{z}}_{k+1}=h\left(\overline{\mathbf{x}}_{k+1}, \mathbf{q}_{k+1}, \dot{\mathbf{q}}_{k+1}, \ddot{\mathbf{q}}_{k+1}, \mathcal{C}_{\mathbf{p}_{1 k+1}}, \ldots,{ }^{\mathcal{C}} \mathbf{p}_{c_{n} k+1}\right),
$$

where $\overline{\mathbf{z}}_{k+1}$ is the predicted measurement vector.

\subsubsection{Update}

The update consists in using the actual measurements to correct, to some extent, the prediction of the previous step. The actual measurement vector is $\mathbf{z}_{k+1}=\left[\begin{array}{lll}\mathbf{y}_{\mathbf{g}_{k+1}}^{\mathbf{t}} & \mathbf{y}_{\mathbf{a} k+1}^{\mathbf{t}} & 0_{1 \times 3 n}\end{array}\right]^{t}$. We define the measurements innovation as

$$
e_{\mathbf{z} k+1}=\mathbf{z}_{k+1}-\overline{\mathbf{z}}_{k+1}
$$

Again, this error is modeled as a Gaussian random variable, and the covariance matrix is approximated as follows:

$$
P_{\mathbf{z} k+1}=H_{k+1} \bar{P}_{k+1} H_{k+1}^{t}+R_{k+1},
$$

where $R_{k+1}$ is the covariance matrix of $\mathbf{w}_{k+1}$, and

$$
H_{k+1}=\frac{\partial h}{\partial \mathbf{x}} \mid \begin{aligned}
& \mathbf{x}=\overline{\mathbf{x}}_{k+1}, \mathbf{q}=\mathbf{q}_{k+1}, \dot{\mathbf{q}}=\dot{\mathbf{q}}_{k+1}, \ddot{\mathbf{q}}=\ddot{\mathbf{q}}_{k+1}, \\
& \mathcal{C}_{\mathbf{p}_{c_{1}}}=\mathcal{C}_{c_{1} k+1}, \ldots, \mathcal{P}_{c_{n}}={ }_{\mathbf{p}_{c_{n} k+1}}
\end{aligned}
$$

This enables us to compute the near-optimal gain $K_{k+1}$ minimizing the quadratic error expectation for the estimation error $\mathbf{e}_{k+1}=\mathbf{x}_{k+1}-\hat{\mathbf{x}}_{k+1}$, where

$$
\hat{x}_{k+1}=\bar{x}_{k+1}+K_{k+1} e_{\mathbf{z} k+1}
$$

The near-optimal gain $K_{k+1}$ is obtained as follows:

$$
K_{k+1}=\bar{P}_{k+1} H_{k+1}^{t} P_{\mathbf{z}}^{-1}
$$

And the corresponding covariance matrix of $\mathbf{e}_{k+1}$ is then

$$
P_{k+1}=\left(I-K_{k+1} H_{k+1}\right) \bar{P}_{k+1}
$$

The size of the measurement vector $\mathbf{z}_{k}$ may change if a contact on environment is removed or added, but this does change neither the state value nor the extended Kalman filter developments.

We have now an estimator of the flexibility deformation in its 6 DoF and its derivatives. This estimation can be directly used for example in order to correct the position of the end effector as we show in the next section. 


\section{HAND POSITION COMPENSATION}

\subsection{The hand position in the world frame}

When a humanoid robot interacts with the environment, the end-effector has usually to follow trajectories in the control reference frame. We have seen that because of flexible parts of the robot, these trajectories may differ in the world reference frame. Moreover, if the robot has to exert forces on the environment in some tasks, such as drilling a wall, the reaction force will create a deformation of the flexible parts and will move the end effector from its reference position.

We propose here to show a direct use of the flexibility observer. We put HRP-2 on its feet, we ask the robot to keep the right hand at a given reference position/orientation, summarized in a homogeneous matrix $M_{r}$, and we push the robot to excite flexibility. However, the hand controller takes only references expressed in $\mathcal{C}$. So, the classical solutions consists in working in the control reference frame $\mathcal{C}$, giving a reference ${ }^{\mathcal{C}} M_{r}=M_{r}$. Obviously the hand will swing with all the robot, and will not keep its reference position, when the flexibility is deformed. Instead, we propose to ask for another reference of hand position and orientation ${ }^{\mathcal{C}} M_{r}=M_{\mathcal{C}}^{-1} M_{r}$, with the flexibility deformation $M_{\mathcal{C}}$ estimated using the extended Kalman filter described in the prevous section. If the flexibility is efficiently reconstructed, the hand will stay at the same position in the world reference frame (see Fig. 4 top left).
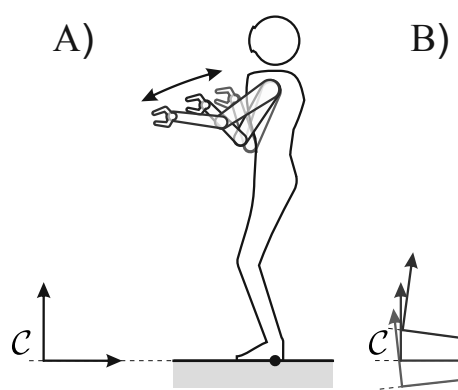

B)
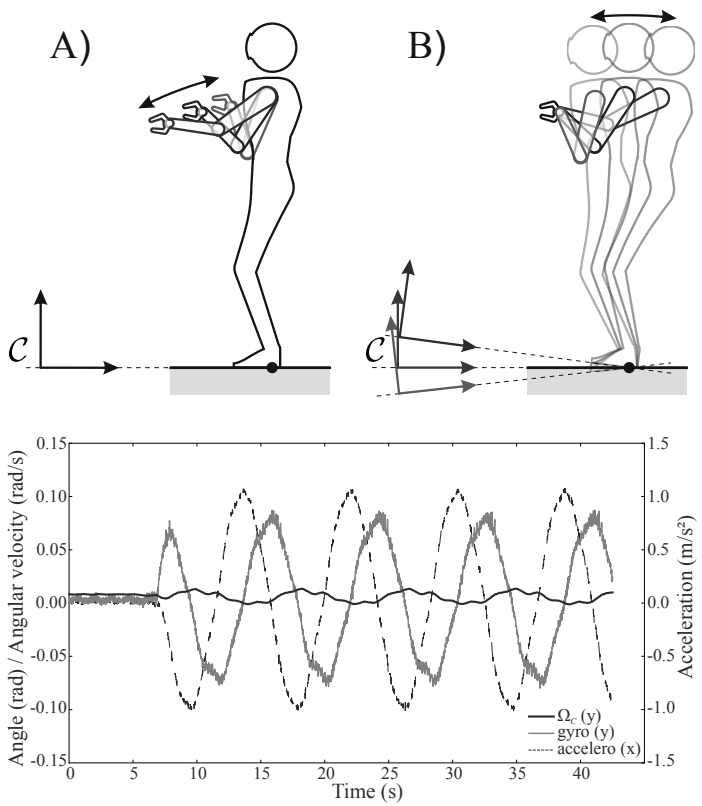
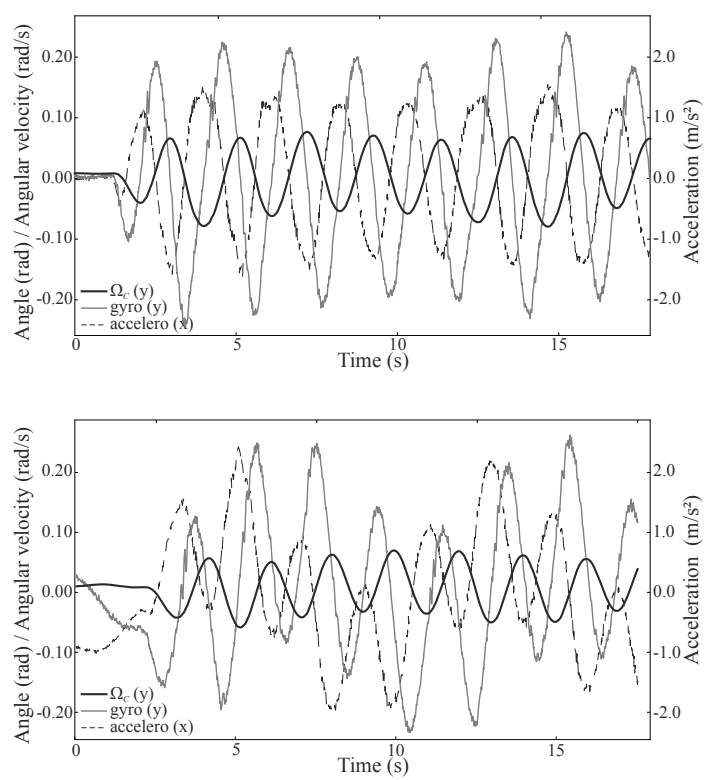

Figure 4: Top left, an illustration of the hand compensation. A) the hand compensation in the world reference frame and B) shows what happens at the same time in the control reference frame. The other figures show the signals of accelerometer along $\mathrm{x}$, the gyrometer around $\mathrm{y}$, and flexibility orientation $\Omega_{\mathcal{C}}$ around $y$, in the case of the three experiments. In top right, the first experiment (the external perturbations on the upright robot). In bottom left, the second experiment (the robot's trunk is oscillating without external perturbations). In bottom right, the third experiment (trunk oscillation and external perturbations are combined).

\subsection{Experimental setting}

To achieve this setting, we use the Stack of Tasks framework[14, 15], which is a task-based hierarchical inverse kinematics solver. The tasks were set, in a decreasing priority order, to (i) keep 
both feet on the ground and the center of mass above the middle of them, in the control reference frame (ii), keep the height ${ }^{\mathcal{C}} \mathbf{p}_{t}$ and the orientation ${ }^{\mathcal{C}} R_{t}$ of the trunk (waist, chest and head), (iii) keep the right hand at ${ }^{{ }^{C}} M_{r}=\mathcal{W}_{M_{\mathcal{C}}}^{-1} M_{r}$ and (iv) reproduce with the left hand the motion of the right hand. The last task is just to play the role of counterweight and avoid unstable dynamical effects of hand compensation.

The stack of tasks (SoT) enables also to introduce a feed-forward term which is the desired velocity of the task in the control reference frame. We introduce then also the following desired hand linear and angular velocities:

$$
\begin{aligned}
\mathcal{C}_{\mathbf{p}_{r}} & =\left[R_{C}^{t} \omega_{C}\right]_{\times} R_{C}^{t}\left(\mathbf{p}_{C}-\mathbf{p}_{r}\right)-R_{C}^{t} \dot{\mathbf{p}}_{C} \\
\mathcal{C}_{\omega_{r}} & =-R_{C}^{t} \omega_{C},
\end{aligned}
$$

where $\mathbf{p}_{r}$ is the reference position of the hand in the world reference frame.

To show the performances of the observer, three experiments are conducted:

- the reference orientation of the trunk ${ }^{\mathcal{C}} R_{t}$ is constant at upright position, and the robot is pushed to excite flexibility. The expected result is that the hand stays at the reference position while the whole robot is displaced.

- the reference orientation of the trunk ${ }^{\mathcal{C}} R_{t}$ oscillates in time. The IMU is then excited and provides oscillating measurements. The flexibility is excited as well, due to torques that move the upper-body, but the excitation is of much smaller magnitude than when the robot is pushed.

- the oscillation of the trunk and the external perturbations are combined. The estimator has to distinguish between the signals due to the oscillation and those due to flexibility deformation.

\subsection{Results}

During the first experiment, the external perturbations made the robot oscillate of up to 0.175 $\operatorname{rad}\left(\right.$ about $\left.10^{\circ}\right)$. The hand position, being at $1.1 \mathrm{~m}$ distance to the contact point, if it was not compensated, would move by about $20 \mathrm{~cm}$. Instead, the hand moves by less than $2.0 \mathrm{~cm}$. During the second experiment, the flexibility was slightly excited and observed by the Kalman filter. However the oscillation of the upper-body created vibrations, detected particularly by the gyrometer, leading to small estimation error, the hand moved by about $1.5 \mathrm{~cm}$. During the third experiment, the combination of oscillation and the perturbation did not degrade the performances and the hand moved still by less than $2.0 \mathrm{~cm}$. The results are summarized in Fig. 5 , and the relevant signals and observations in Fig. 4. The video of the experiments[16] presents also the performances under several angles, including the ankle of the robot, and shows responses to lateral perturbations. We remind that only the right hand is stabilized and the left hand is just a counterweight.

\section{FLEXIBILITY STABILIZATION}

\subsection{Balance and flexibility}

Bipedal locomotion enables us to move through various kinds of environments, because it relies on small contact surfaces. However, this property requires the walking system to be carefully balanced, because the dynamics of the whole upright body only depends on the forces at these contacts[6]. Several criteria were developed to ensure balance of biped robots. The most prominent criterion is the ability to keep a point on the ground called zero moment point (ZMP) or center of pressure (CoP) strictly inside the convex hull of supporting surfaces[17]. This criterion is usually guaranteed by a controller which generates trajectories of feet and center of mass (CoM) [18].

However, when the robot structure contains flexible parts, the compliance modifies the configuration of the whole robot, including its center of mass. This modification occurs not only on 

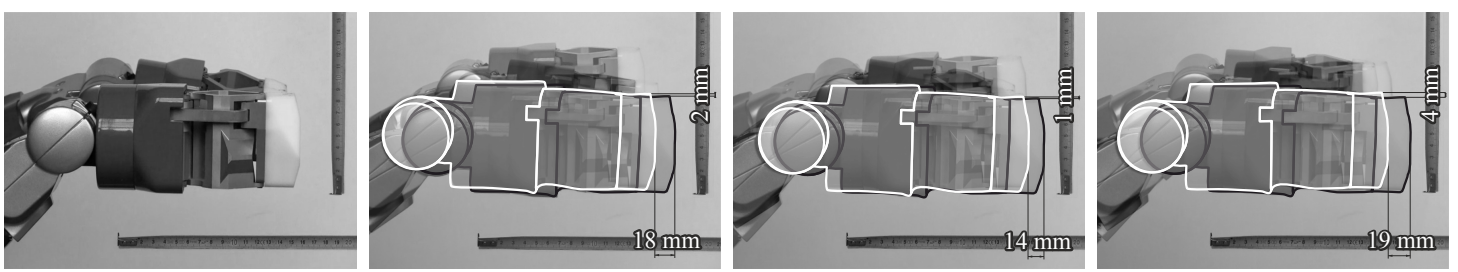

Figure 5: From left to right: (i) a photo of the robot's hand during the compensation experiment. (ii) A superimposition of the two pictures of the extreme points of hand stabilization during the excitation of the flexibility. (iii) A superimposition of extreme positions during oscillation. And (iv) a superimposition of extreme positions during combined oscillation and excitation of the flexibility. For each superimposition, the position displacement for the hand is shown.

the position of the CoM, but also on its velocity and acceleration in the world reference frame. However, the position and acceleration of the CoM are the most important parameters to describe the $\mathrm{CoP}$ of the humanoid robot. Therefore if the flexibility dynamics exceeds some threshold, even if it is only in acceleration, the center of pressure may leave the safe area, and lead the robot to flip and fall.

Furthermore, it is not sufficient to reconstruct only the CoM motion in the world reference frame to guarantee balance. Indeed, depending on the compliance response to deformation, contact forces may be modified, even for the same CoM position. Fig. 6 shows an example of two inverted pendulums with torsional elasticity at contact having the same CoM position but with completely different contact forces. Therefore, this property may lead the robot fall because the CoP position is entirely defined by contact forces. Moreover, an elasticity of the compliance will store energy. If inappropriate frequencies appear in the control of the robot, the spring will resonate and conduce to unstable and dangerous kinematics.
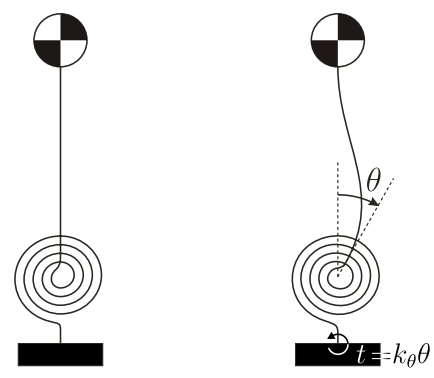

Figure 6: On the left, An inverted pendulum with elastic torsion at rest position. On the right, the elasticity is not at rest position generating a torque $t$ at contact point. This difference occurs even if the center of mass position is the same for both pendulums.

Nevertheless, if we are able to rebuild reliably the robot kinematics and the flexibility state, we can correct the motion of the robot to minimize the energy stored in the elasticity and to improve the tracking of the CoM reference position in the world reference frame. We call stabilization the robot control with this two wise objective. We show next a demonstration of the possibility to design a stable closed-loop stabilizer that takes as state feedback the estimation of the observer presented in Section 3.

\subsection{A model of flexibility}

The main purpose of a flexibility-deformation state-observer should go beyond simply stabilizing end-effectors. Indeed, estimating efficiently the flexibility deformation means that (i) we know the 
actual kinematics of the center of mass in the world reference frame and (ii) we have an estimation of the ground reaction forces if we have a model of the force-response to deformations of the flexible part of the robot. Both estimations are precious information on the dynamic balance of the humanoid robot. Therefore, the main long term objective of this study is to open the way to balancing and stabilizing techniques that could run on robots that are not equipped with force sensors and to improve the estimation quality of robots that do have these sensors.

The example of Fig. 6 shows clearly that the kinematics of the CoM by itself cannot be enough to estimate the balance state of the robot. Contact forces have to be estimated to make sure the feet are not about to flip because of the deviation of the $\mathrm{CoP}$ due to deformation reaction forces. The force response of flexibility deformation has to be modeled.

To emphasize on the quality of observation, we take the simplest imaginable model of HRP-2 with its flexible bush. The model is an inverted pendulum with the mass of the robot concentrated at the CoM position. We decouple the rotation around $\mathbf{y}$ axis from the rotation around $\mathbf{x}$ axis. The deformation is simplified then with two angles $\theta_{y}$ and $\theta_{x}$ respectively. The model boils down to two 2D inverted pendulums. We consider the CoM height as constant in the control frame, similarly to the cart-table model except that the flexibility may modify the CoM height in the world frame. Then we control separately the CoM acceleration along $\mathbf{x}$ and $\mathbf{y}$ separately.

Without loss of generality, we show the dynamic model of the single 2D pendulum related to the $\mathbf{x}$ axis, and we will apply this model and the outcoming control on both axes. The model is described in Fig. 7. The flexibility is a simple angle $\theta$ which rotates the control reference frame relative to the world frame. The center of rotation is the contact point at $(0,0)$ of both frames. The position of the CoM in the control frame is $(\xi, \zeta)$ and in the world frame is $(x, z)$ such that :

$$
x=\xi \cos \theta-\zeta \sin \theta \text { and } z=\xi \sin \theta+\zeta \cos \theta
$$

By considering that $\zeta$ is constant, this leads to

$$
\begin{aligned}
& \dot{x}=\dot{\xi} \cos \theta-\dot{\theta} z \quad \text { and } \dot{z}=\dot{\xi} \sin \theta+\dot{\theta} x \\
& \ddot{x}=\ddot{\xi} \cos \theta-2 \dot{\theta} \dot{z}+\dot{\theta}^{2} x-\ddot{\theta} z \quad \text { and } \ddot{z}=\ddot{\xi} \sin \theta+2 \dot{\theta} \dot{x}+\dot{\theta}^{2} z+\ddot{\theta} x
\end{aligned}
$$

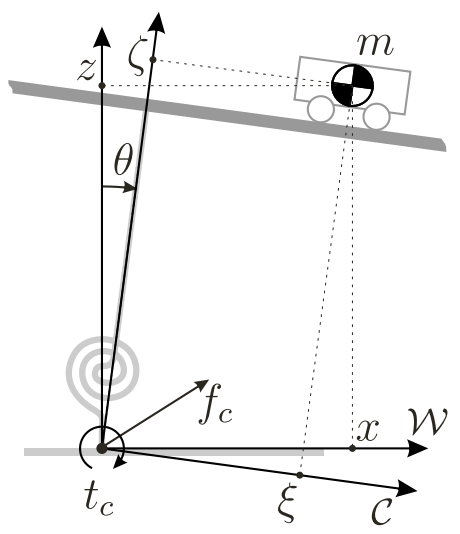

Figure 7: Our model of the flexibility. It is a cart table model of mass $m$ defined in control reference frame, which is moved by the flexibility consisting in an angle $\theta$. The center of mass is represented by the center of the cart. Its position is $(x, z)$ in the world frame and $(\xi, \zeta)$ in the control frame. The contact force and torques are denote $f_{c}$ and $t_{c}$ respectively.

The dynamics of this system is described by

$$
\begin{aligned}
f_{c, x} & =m \ddot{x} \\
f_{c, z} & =m\left(\ddot{z}+g_{0}\right) \\
t_{c} & =z f_{c, x}-x f_{c, z},
\end{aligned}
$$


leading to

$$
\begin{aligned}
t_{c} & =m\left(z \ddot{x}-x \ddot{z}-x g_{0}\right) \\
& =m\left(z\left(\ddot{\xi} \cos \theta-2 \dot{\theta} \dot{z}+\dot{\theta}^{2} x-\ddot{\theta} z\right)-x\left(\ddot{\xi} \sin \theta+2 \dot{\theta} \dot{x}+\dot{\theta}^{2} z+\ddot{\theta} x\right)-x g_{0}\right) \\
& =m\left(\ddot{\xi}(z \cos \theta-x \sin \theta)-2 \dot{\theta}(z \dot{z}+x \dot{x})-\ddot{\theta}\left(z^{2}+x^{2}\right)-x g_{0}\right)
\end{aligned}
$$

We model the flexibility as a relation between the angle $\theta$ and the torque $t_{c}$. The simplest possible model is the rotational spring: $t_{c}=k_{\theta} \theta$ where $k_{\theta}$ is the stiffness of the spring. Therefore Eq. (35) provides us with the following flexibility dynamics

$$
\ddot{\theta}=\frac{m\left(\ddot{\xi}(z \cos \theta-x \sin \theta)-2 \dot{\theta}(x \dot{x}+z \dot{z})-x g_{0}\right)-k_{\theta} \theta}{m\left(x^{2}+z^{2}\right)}
$$

This relation together with Eq. (29) and the straightforward dynamics of $x, \dot{x}, z$ and $\dot{z}$ constitute the dynamic model of the system.

We denote now by $\mathbf{s}=(x \theta \dot{x} \dot{\theta})^{t}$ the state vector and $\mathbf{u}=(\ddot{\xi})$ the control vector. For our demonstration, we show the stabilization of the robot in stance position on both parallel feet. We consider as a desirable state of the robot the position where the center of mass above the origin and the flexibility is still, i.e. $x=\dot{x}=\theta=\dot{\theta}=\ddot{\zeta}=0$ and $z=\zeta$. We assume that the flexibility angle remains small, therefore the described dynamics can be approximated by linearization around this desired equilibrium: $\dot{\mathbf{s}}=A \mathbf{s}+B \mathbf{u}$, with

$$
A=\left(\begin{array}{cccc}
0 & 0 & 1 & 0 \\
0 & 0 & 0 & 1 \\
\frac{g_{0}}{\zeta} & \frac{k_{\theta}}{m \zeta} & 0 & 0 \\
\frac{-g_{0}}{\zeta^{2}} & \frac{-k_{\theta}}{m \zeta^{2}} & 0 & 0
\end{array}\right) \quad B=\left(\begin{array}{c}
0 \\
0 \\
0 \\
\frac{1}{\zeta}
\end{array}\right)
$$

The controllability matrix

$$
\left[\begin{array}{llll}
B & A B & A^{2} B & A^{3} B
\end{array}\right]=\left(\begin{array}{cccc}
0 & 0 & 0 & \frac{k_{\theta}}{m \zeta^{2}} \\
0 & \frac{1}{\zeta} & 0 & \frac{-k_{\theta}}{m \zeta^{3}} \\
0 & 0 & \frac{k_{\theta}}{m \zeta^{2}} & 0 \\
\frac{1}{\zeta} & 0 & \frac{-k_{\theta}}{m \zeta^{3}} & 0
\end{array}\right)
$$

is defined and full rank as long as $\zeta$ and $k_{\theta}$ are positive.

An interesting note is that the described system can be seen as a forth order linear system to control the CoM position in the world frame. With this consideration, the control boils down to a snap control. ${ }^{2}$ Using a linear quadratic regulator (LQR) we obtain the optimal control vector $\mathbf{u}$ which minimize the angle $\theta$ and the position error in CoM position $x$.

\subsection{Stabilization of HRP-2}

We apply the control described earlier on HRP-2. The flexibility stiffness around $\mathbf{y}$ axis was identified[19] to be roughly around $k_{\theta, \mathbf{y}}=500 \mathrm{Nm} / \mathrm{rad}$. The elasticity around $\mathbf{x}$ axis is only due to the small linear compliance of the bushes since the robot is on its both feet. Therefore, the equivalent angular spring has a very high stiffness. We set it to $k_{\theta, \mathbf{x}}=50000 \mathrm{Nm} / \mathrm{rad}$ and around $\mathbf{x}$ axis. The state feedback of each axis is a part of the estimation vector provided by the state observer implemented earlier: the angle $\theta$ and its time-derivative are the components of vectors $\Omega$ and $\omega$, and the positions of the CoM in the global frame are simply obtained by transformation from its known position in the control frame using also the estimation of the flexibility. We use the stack of tasks framework [15] to generate the joint motion to track reference positions of the CoM.

\footnotetext{
${ }^{2}$ The snap is the fourth order time-derivative of the position, i.e. the time-derivative of the jerk
} 
The experiment is divided in two steps: (i) the robot is standing and receives external pushes, (ii) the reference position of the CoM is modified discontinuously $1 \mathrm{~cm}$ forward and after few seconds $1 \mathrm{~cm}$ backward. Both experiments are performed with and without stabilizer.

We restricted the results on rotations around $\mathbf{y}$ axis, because it has much more amplitude and there can be significant improvement compared to a rigid robot. The results are shown in Fig. 8 . The stabilizer provides much more reduced dynamics of the flexibility and lower convergence time. What is remarkable is the fact that the state feedback did not use force sensors, and does not have any model of the forces, it gives no prediction on the real dynamics of the system, but it still provides the controller with reliable estimation enabling stable control of the CoM and the flexibility. The video of this experiment [16] shows all these presented results including sideways perturbations. It demonstrates clearly the benefits of the stabilizer.
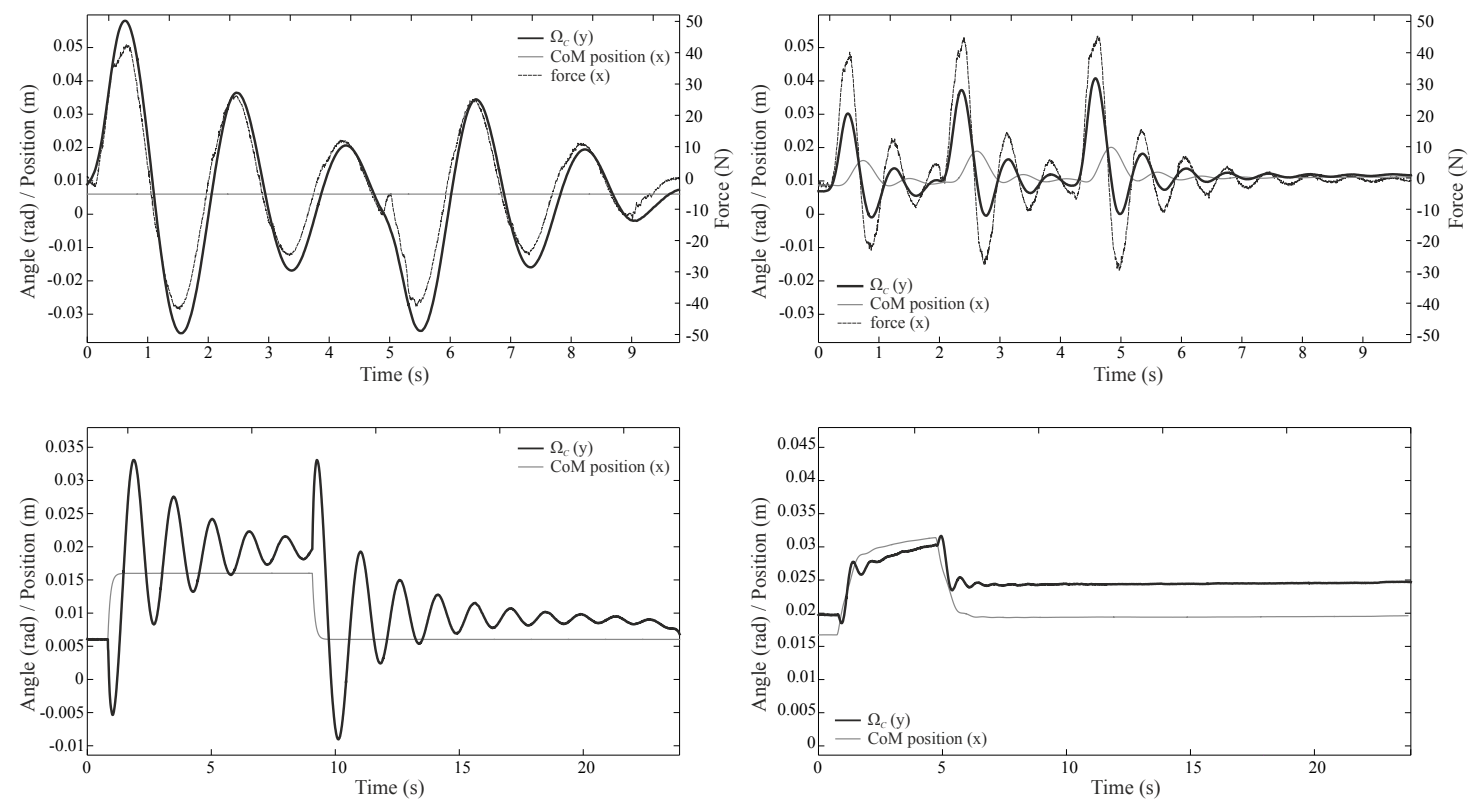

Figure 8: These four figures show over time the flexibility state around $\mathbf{y}$ axis and the position of the CoM in the control frame (denoted $\xi$ in the model described earlier). The two top figures show the responses the first experiment (external pushes), without stabilizer (left) and with stabilizer (right). Additionally, there is a plot of measured linear forces along $\mathbf{x}$ axis at feet. This force is measured with force sensor, so we do not use it to stabilize the robot, we display it to show that the pushing forces are comparable between both cases. In the bottom figures, we show the second experiment (discontinuous steps in the reference position of the CoM) without (left) and with stabilizer (right). In both cases, the stabilizer guarantees lower amplitudes of the flexibility and much faster convergence to stationary state.

The performances of the stabilizer are obviously not perfect. They are not sufficient to enable HRP-2 to walk. But this controller is the simplest possible implementation of a flexibility stabilizer, and we intended use it as a proof of concept of the reliability of our flexibility estimation, and the relevance of the observer formulation.

\section{DISCUSSION AND CONCLUSION}

We have seen through this paper that the contact with the environment enables us to take profit from the position-orientation coupling, in order to better distinguish between body accelerations and gravitational component in accelerometer measurements. The separation of these two signals 
have two benefits: it increases the precision in verticality estimation (pitch and roll), but also it provides information on the acceleration of the attitude and position. This estimation allows to compensate for the displacement of the end effector due to the deformation, and even to give a state feedback to a stable closed-loop flexibility and CoM stabilizer.

The combination of the inertial measurements and legs kinematics is already a subject of active research, but almost exclusively on multi-legged robots for odometry and localization purposes[20, $21,22,23,24,25]$. These methods provide reliable information on the position in the world. However, none of them took profit of the accelerations-gravity decoupling, even when attitude reconstruction was an important component of the reconstructed state vector[24, 25].

To our best knowledge, only one method takes this advantage, which is presented by Bloesch et al.[26, 27] for a multi-legged robot. They used the contact information provided by proprioceptive sensors and kinematics model and merged them with IMU signals in a multiplicative/additive extended Kalman filter. They constrained the dynamics of the state to respect contact kinematics, and use translation-rotation coupling to improve their estimation. However, their model considers the IMU signals as inputs to the system and not measurements. So their model of the dynamics is an integration of the measurements. This prevents having another model of the state dynamics (e.g. inverted pendulum, spring damper, etc) because this would remove IMU information from models. They also consider slipping contacts as measurements, which may be adapted to walk on uncertain or slippery environments, but which leads to the non-observability of the position as aknowledged by the authors. That means that the position may drift for long observation periods. This would lead for example the hand compensation experiment to deviate eventually from the reference positions. This issue could possibly be partially solved by reducing the covariance of the noise model of the measurements, but Kalman filtering is likely to have numerical issues with too certain or perfect measurements[28]. In fact, for humanoid robots in known environments, we can consider the contacts as firmly linked to the ground and this should be exploited to constrain the dynamics of the reconstructed attitude as presented in our study. Finally, Bloesch et al. model a rigid contact with the ground while our model enables small deviation in translation from the contact point. This deviation may happen in the case of a flexibility in translation, but this is different from slipping contact because in our case average contact position is assumed constant which it is guaranteed by our observer.

Some issues may arise and be considered as limitations of our approach. First, if there is more than one flexible part in the robot, one single IMU does not guarantee the observability of the flexibility. In that case, a solution may be to use several IMUs[29]. Second, in the case the joint positions are not perfectly known, the estimation errors would be proportional to errors in contact positions and to errors in the position/orientation of the IMU and their derivatives in the control frame.

Finally, we do not believe that the presented flexibility observer has reached its optimal potential performances. We have seen in the design of the stabilizer that we can build a model of the force response to flexibility deformation. This may provide us with a better state predictor than the simple double integrator. If the position estimation is reliable and the model good enough, the estimated forces will enable a much cleaner prediction of the velocity and especially the acceleration components of the flexibility vector. Furthermore, this model will provide us with force measurements, which is precisely what is missing in our scheme. This is already in our ongoing research[30]. This simulated measurement can be used to achieve much better stabilizers and even if the robot has already force sensors, they can be used as a new feedback to the observer in order to improve again the estimation.

\section{References}

[1] N. Kanehira, T. Kawasaki, S. Ohta, T. Ismumi, T. Kawada, F. Kanehiro, S. Kajita, and K. Kaneko. Design and experiments of advanced leg module (hrp-2l) for humanoid robot (hrp-2) development. In Intelligent Robots and Systems, volume 3, 2002. 
[2] S. Kajita, K. Yokoi, M. Saigo, and K. Tanie. Balancing a humanoid robot using backdrive concerned torque control and direct angular momentum feedback. In Intl. Conf. Robotics and Automation, 2001.

[3] S. Kajita, T. Nagasaki, K. Kaneko, K. Yokoi, and K. Tanie. A running controller of humanoid biped hrp-2lr. In Intl. Conf. Robotics and Automation. IEEE, 2005.

[4] R. M Voyles, J. D. Morrow, and P. K Khosla. The shape from motion approach to rapid and precise force/torque sensor calibration. Journal of dynamic systems, measurement, and control, 119(2):229-235, 1997.

[5] Aldebaran Robotics. Romeo. http://www.projetromeo.com, 2011.

[6] P.-B. Wieber. On the stability of walking systems. In Proceedings of the International Workshop on Humanoid and Human Friendly Robotics, Tsukuba, Japan, 2002.

[7] N. Perrin, N. Tsagarakis, and D. G. Caldwell. Compliant attitude control and stepping strategy for balance recovery with the humanoid coman. In Intelligent Robots and Systems (IROS), 2013, pages 4145-4151. IEEE, 2013.

[8] S. Kajita, M. Morisawa, K. Miura, S. Nakaoka, K. Harada, K. Kaneko, F. Kanehiro, and K. Yokoi. Biped walking stabilization based on linear inverted pendulum tracking. In Intelligent Robots and Systems (IROS), pages 4489-4496, Oct 2010.

[9] J. L. Crassidis, F. L. Markley, and Y. Cheng. Survey of nonlinear attitude estimation methods. Journal of Guidance, Control, and Dynamics, 30(1):12-28, 2007.

[10] V. Kubelka and M. Reinstein. Complementary filtering approach to orientation estimation using inertial sensors only. In Robotics and Automation (ICRA), May 2012.

[11] B. Barshan and H. F Durrant-Whyte. Inertial navigation systems for mobile robots. Robotics and Automation, IEEE Transactions on, 11(3):328-342, 1995.

[12] P.-C. Lin, H. Komsuoglu, and D. E Koditschek. Sensor data fusion for body state estimation in a hexapod robot with dynamical gaits. Robotics, IEEE Transactions on, 22(5):932-943, 2006.

[13] O. Gür. and U. Saranli. Model-based proprioceptive state estimation for spring-mass running. In Proceedings of Robotics: Science and Systems, 2011.

[14] N. Mansard, O. Stasse, P. Evrard, and A Kheddar. A versatile generalized inverted kinematics implementation for collaborative working humanoid robots: The stack of tasks. In International Conference on Advanced Robotics, pages 1-6, June 2009.

[15] The Stack of Tasks Framework. Open source project availabe online at https://github.com/stack-of-tasks.

[16] The video of all the experiments on HRP-2 robot. Availabe online at http://mehdi.benallegue.com/ijhr2015video or https://youtu.be/_xpEIPgy8M0.

[17] M. Vukobratović and B. Borovac. Zero-moment point - thirty five years of its life. International Journal of Humanoid Robotics, 1(01):157-173, 2004.

[18] S. Kajita, F. Kanehiro, K. Kaneko, K. Fujiwara, K. Harada, K. Yokoi, and H. Hirukawa. Biped walking pattern generation by using preview control of zero-moment point. In International Conference on Robotics and Automation, 2003.

[19] Y. Mikami, T. Moulard, E. Yoshida, and G. Venture. Identification of HRP-2 foot's dynamics. In International Conference on Intelligent Robots and Systems, 2014. 
[20] B. Gassmann, F. Zacharias, J.M. Zollner, and R. Dillmann. Localization of walking robots. In IEEE International Conference on Robotics and Automation, 2005.

[21] S. Chitta, P. Vernaza, R. Geykhman, and D.D. Lee. Proprioceptive localilzatilon for a quadrupedal robot on known terrain. In IEEE International Conference onRobotics and Automation, pages 4582-4587, April 2007.

[22] J.A. Cobano, J. Estremera, and P. Gonzalez de Santos. Location of legged robots in outdoor environments. Robotics and Autonomous Systems, 56(9):751 - 761, 2008.

[23] P.-C. Lin, H. Komsuoglu, and D.E. Koditschek. Sensor data fusion for body state estimation in a hexapod robot with dynamical gaits. Robotics, IEEE Transactions on, 22(5):932-943, Oct 2006 .

[24] A Chilian, H. Hirschmuller, and M. Gorner. Multisensor data fusion for robust pose estimation of a six-legged walking robot. In IEEE/RSJ International Conference on Intelligent Robots and Systems (IROS), pages 2497-2504, Sept 2011.

[25] M. Reinstein and M. Hoffmann. Dead reckoning in a dynamic quadruped robot: Inertial navigation system aided by a legged odometer. In Robotics and Automation (ICRA), 2011 IEEE International Conference on, pages 617-624, May 2011.

[26] M. Bloesch, M. Hutter, M. Hoepflinger, S. Leutenegger, C. Gehring, C. D. Remy, and R. Siegwart. State estimation for legged robots - consistent fusion of leg kinematics and IMU. In Proceedings of Robotics: Science and Systems, 2012.

[27] N. Rotella, M. Bloesch, L. Righetti, and S. Schaal. State estimation for a humanoid robot. In Intelligent Robots and Systems (IROS 2014), 2014 IEEE/RSJ International Conference on, pages 952-958, Sept 2014.

[28] Dan Simon and Tien Li Chia. Kalman filtering with state equality constraints. Aerospace and Electronic Systems, IEEE Transactions on, 38(1):128-136, 2002.

[29] S. Traversaro, D. Pucci, and F. Nori. In situ calibration of six-axes force torque sensors using accelerometer measurements. arXiv preprint arXiv:1410.0885, 2014.

[30] Alexis Mifsud, Mehdi Benallegue, and Florent Lamiraux. Estimation of Contact Forces and Floating Base Kinematics of a Humanoid Robot Using Only Inertial Measurement Units. Submitted to IROS2015, 2015. available at https://hal.archives-ouvertes.fr/hal-01142399. 


\section{Acknowledgements}

This work was partially funded by the French project Romeo-2 and the project ERC Advanced Grant 340050 Actanthrope.

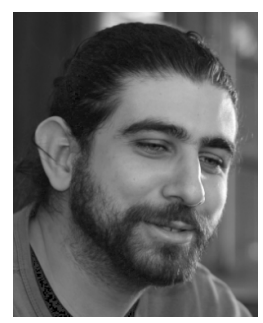

Mehdi Benallegue received the ingénieur degree from the Institut National d'Informatique (INI), Algeria, in 2007, the M.Sc. degree from the University of Paris 7, Paris, France, in 2008, and the Ph.D. degree from Université de Montpellier 2, France, in 2011. He is currently a Research Associate with the Laboratory for Analysis and Architecture of Systems, Toulouse, France. His research interests include humanoid robots, state observers, locomotion, imitation, learning, and computational geometry.

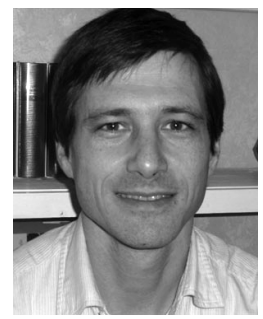

Florent Lamiraux received the Graduate degree from the Ecole Polytechnique Paris, Paris, France, in 1993 and the Ph.D. degree in computer science from the Institut National Polytechnique de Toulouse, Toulouse, France, in 1997 for his research on mobile robots. He was with Rice University, Houston, TX, as a Research Associate for two years. He is currently a Directeur de Recherche with

LAAS-CNRS, Université de Toulouse, where he is involved with humanoid robots. 